\title{
Holter Monitoring (Ambulatory Electrocardiography) Defined Cardiac Arrhythmia Among Patients Presented with Palpitation in the Primary Care Setting
}

\author{
Lap-kin Chiang*, Lorna Ng \\ Family Medicine and General Outpatient Department, Kwong Wah Hospital, Hong Kong Special Administrative Region, China
}

\author{
Email address: \\ chialk@ha.org.hk (Lap-kin Chiang),ngl@ha.org.hk (Lorna Ng) \\ ${ }^{*}$ Corresponding author
}

\section{To cite this article:}

Lap-kin Chiang, Lorna Ng. Holter Monitoring (Ambulatory Electrocardiography) Defined Cardiac Arrhythmia Among Patients Presented with Palpitation in the Primary Care Setting. Journal of Family Medicine and Health Care. Vol. 3, No. 1, 2017, pp. 12-16. doi: $10.11648 /$ j.jfmhc.20170301.13

Received: December 13, 2016; Accepted: December 24, 2016; Published: January 18, 2017

\begin{abstract}
Palpitation is non-specific and represents one of the most common symptoms in general medical settings. Discerning cardiac from noncardiac causes is important. A 24-hour ambulatory electrocardiography (Holter) monitor is usually used. This is a retrospective cross-sectional review including all patients presented with palpitation and had Holter monitoring performed in a regional primary care clinic of Hong Kong during the year 2010 to 2014. Clinical information and Holter outcomes were retrieved and analyzed to examine Holter monitoring defined significant cardiac arrhythmia and assess the predictive patient characteristics associated with significant cardiac arrhythmia. Holter monitoring were arranged for $89(31.9 \%)$ male and $190(68.1 \%)$ female patients. $163(58.4 \%)$ patients had associated chronic comorbidity, including 38.0\% had hypertension. 109 (39.1\%) Holter monitoring showed significant cardiac arrhythmia, including prolong QT interval (11.1\%), frequent supraventricular/ventricular ectopics $(9.0 \%)$ and supraventricular/ventricular ectopics in bigeminy or trigeminy $(5.0 \%)$. Patients who were smokers, or with concomitant ischaemic heart disease were more likely to have significant cardiac arrhythmia $(\mathrm{P}<0.05) .39 .1 \%$ of Holter monitoring for patients presented with palpitation in the primary care setting have significant cardiac arrhythmia, which need referral to medical specialist for further management.
\end{abstract}

Keywords: Electrocardiography, Holter Monitoring, Palpitation, Primary Care

\section{Introduction}

Palpitations are non-specific and represent one of the most common symptoms in general medical settings, reported by as many as $16 \%$ of outpatient patients. [1] Establishing the cause of palpitation may be difficult because historical clues are not always accurate. [2] However, discerning cardiac from noncardiac causes is important, given the potential risk of sudden death in those with an underlying cardiac etiology. [3] A 24-hour ambulatory electrocardiography (AECG or Holter) monitor is usually used, aiming for a higher yield of detection of possible cardiac arrhythmia, and its clinical efficacy in this aspect has been well established. [4]

American Heart Association and American College of Cardiology (ACC/AHA), recommend using Holter to assess symptoms suggestive of underlying cardiac rhythm disturbance, including unexplained recurrent palpitation, unexplained syncope, near syncope, or episodic dizziness where the cause is not obvious. [4] In primary care setting, the focus of using Holter is for early detection of possible life-threatening cardiac arrhythmia as a cause of symptoms. It can minimize the risk of patients by shortening the time to diagnosis and initiate appropriate early referring to specialist care.

The Family Medicine and General Outpatient Department (FM\&GOPD) of Kwong Wah Hospital is a primary care clinic affiliated to a regional hospital of Hong Kong. The attending primary care doctor can book Holter monitoring directly at Cardiac Unit of the hospital. This retrospective cross-sectional review will recruit all Holter monitorings ordered by FM\&GOPD doctors for patients presented with palpitation and assess the patient characteristics associated with significant cardiac arrhythmias. 


\section{Method}

\subsection{Objectives}

I) To examine outcomes of Holter monitoring performed for patients presented with palpitation in a primary care clinic.

II) To determine patient characteristics associated with significant cardiac arrhythmias.

\subsection{Study Population}

All Holter monitoring performed during the period $1^{\text {st }}$ January 2010 to $31^{\text {st }}$ December 2014 in the Kwong Wah Hospital FM\&GOPD were retrieved. (Refer to Figure 1) Patient clinical details including demographics, presenting symptoms and Holter results were collected. Those patients presented with primary complaint of palpitation were included for further analysis.

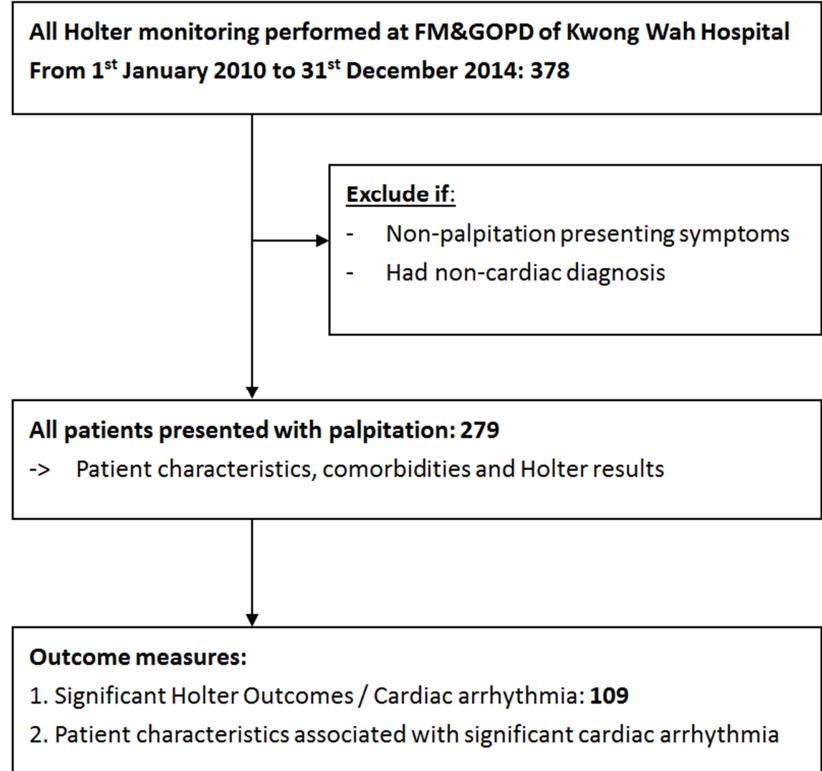

Figure 1. Study Flow Chart.

\subsection{Sample Size Estimation}

Sample size was calculated on an assumption prevalence of Holter monitoring defined significant cardiac arrhythmia in the primary care setting of $15 \%$ with $5 \%$ margins of error and $95 \%$ confidence level. The calculated sample size is 194 . [5] Therefore, the study needs at least 194 patients with eligible Holter monitoring reports.

\subsection{Definition and Holter Monitoring}

Palpitation was defined as one or more of the following complaints: fast heart beats, skipped heart beats, irregular heart rate and heart fluttering, racing or pounding. [6] "New onset" palpitation referred to patient's symptom(s) had occurred within 3 months preceding the first visit to the clinic. Patient who had a history of previous episodes of palpitation or palpitation had occurred more than 3 months was defined as "old" symptom.

All Holter monitoring were performed by the Cardiac Unit at Kwong Wah Hospital, and standardized reports verified by authorized Cardiac Unit doctors were generated and sent to the referring primary care physicians. Content of the reports included patient demographic data, heart rate data and variability, QT analysis, ST episode analysis, pacer analysis, ventricular ectopy, supraventricular ectopy and atrial fibrillation.

The Holter outcome classification in this study was dichotomous (significant cardiac arrhythmia versus insignificant cardiac arrhythmia or normal) to reflect the reality of primary care decision making. [6] All diagnosis of significant cardiac arrhythmia will be referred to medical specialist for further evaluation and management.

The following definitions for various Holter abnormalities were used:

- Frequent ectopy: $>15 / 1,000$ atrial or ventricular ectopic beats. [7]

- Significant pause: RR interval of $\geqq 2.8$ seconds. [7]

- Paroxysmal atrial fibrillation (PAF): an irregular rhythm without P-wave activity sustained for $\geqq 10$ beats. [7]

- Prolong QT interval: corrected QT interval (QTc) $>440$ msec for men and $>460$ msec for women. $[8,9]$

- Bigeminy: pairs of normal and premature complex. [10]

- Trigeminy: a premature complex that follows two normal beats. [10]

The following were considered to be significant Holter findings: ventricular tachycardia (VT), paroxysmal supraventricular tachycardia (PSVT), atrial fibrillation (AF, including paroxysmal atrial fibrillation, PAF), atrial flutter, atrial tachycardia, supraventricular or ventricular ectopy (SVE or VE) occurring in couplets or having a multifocal origin, high grade atrioventricular (AV) block, i.e. second degree or third degree, sick sinus syndrome (SSS), prolong QT interval, or significant change in ST segment. $[6,11]$ Insignificant cardiac arrhythmia encompassed patients with non-pathological atrial or ventricular ectopy, e.g. sporadic, occasional unifocal ectopy. [6]

\subsection{Statistical Analysis}

Descriptive statistics including mean, standard deviation, frequency and percentages will be used to summarize the baseline characteristics and outcome measures. Chi-square test and multivariate analysis with logistic regression were used to assess the patient characteristics associated with significant cardiac arrhythmia. All analyses were conducted using Statistical Package for the Social Sciences version 21 (SPSS Inc., United States).

\subsection{Research Ethics}

The study was approved by Hong Kong Hospital Authority Kowloon West Cluster Research Ethics Committee.

\section{Results}

In the period from $1^{\text {st }}$ January 2010 to $31^{\text {st }}$ December 2014, 378 Holter monitoring were arranged by the primary care 
doctors of FM\&GOPD. 279 Holter monitoring were performed for $89(31.9 \%)$ male and $190(68.1 \%)$ female patients presented with palpitation. Their mean (SD) age were 60.4 (15.2) and $57.6(13.8)$ years old respectively. $57.3 \%$ had new onset of palpitation of less than three months preceded the first relevant consultation. The demographic characteristics of patients were summarized in Table $1.58 .4 \%$ patients had at least one concomitant chronic comorbidity, while the commonest condition was obesity $(48.0 \%)$.

Table 1. Patient demographics and characteristics associated with significant Holter outcomes.

\begin{tabular}{|c|c|c|c|c|c|}
\hline & Frequency $(\%)$ & Sig. Outcome & $\mathrm{X}^{2}$ test, $p$-value & OR & $95 \% \mathrm{CI}$ \\
\hline Study population: & 279 & $109(39.1 \%)$ & & & \\
\hline \multicolumn{6}{|l|}{ Gender: } \\
\hline Female & $190(68.1 \%)$ & $72(37.9 \%)$ & - & 1 & - \\
\hline Male & $89(31.9 \%)$ & $37(41.6 \%)$ & 0.557 & 1.17 & $0.70-1.95$ \\
\hline Smoker (Ex- or current) & $57(20.4 \%)$ & $29(50.9 \%)$ & 0.041 & 1.84 & $1.02-3.31$ \\
\hline \multicolumn{6}{|l|}{ Age distribution: } \\
\hline$</=40$ & $28(10.0 \%)$ & $12(42.9 \%)$ & - & 1 & - \\
\hline $41-60$ & $125(44.8 \%)$ & $35(28.0 \%)$ & 0.123 & 0.52 & $0.22-1.21$ \\
\hline $61-80$ & $115(41.2 \%)$ & $53(46.0 \%)$ & 0.758 & 1.14 & $0.50-2.62$ \\
\hline$>80$ & $11(4.0 \%)$ & $9(81.8 \%)$ & 0.028 & 6.00 & $1.09-33.02$ \\
\hline No chronic comorbidity: & $116(41.6 \%)$ & $39(33.6 \%)$ & - & 1 & - \\
\hline Chronic comorbidity $^{*}$ & $163(58.4 \%)$ & $70(42.9 \%)$ & 0.116 & 1.49 & $0.91-2.44$ \\
\hline Hypertension & $106(38.0 \%)$ & $48(45.3 \%)$ & 0.096 & 1.52 & $0.93-2.49$ \\
\hline Diabetes mellitus & $26(9.3 \%)$ & $10(38.5 \%)$ & 0.947 & 0.97 & $0.42-2.23$ \\
\hline$I F G / I G T$ & $17(6.1 \%)$ & $9(52.9 \%)$ & 0.226 & 1.82 & $0.68-4.88$ \\
\hline Dyslipidaemia & $43(15.4 \%)$ & $20(46.5 \%)$ & 0.277 & 1.44 & $0.75-2.76$ \\
\hline Ischaemia heart disease & $8(2.9 \%)$ & $7(87.5 \%)$ & 0.004 & 11.60 & $1.41-95.63$ \\
\hline CVA/TIA & $5(1.8 \%)$ & $3(60 \%)$ & 0.333 & 2.38 & $0.39-14.47$ \\
\hline Obesity & $134(48.0 \%)$ & $60(44.8 \%)$ & 0.060 & 1.59 & $0.98-2.58$ \\
\hline New onset & $160(57.3 \%)$ & $58(36.3 \%)$ & 0.263 & 0.76 & $0.47-1.23$ \\
\hline
\end{tabular}

$I F G$ /IGT: Impaired fasting glucose /Impaired glucose tolerance; CVA/TIA: Cerebral vascular accident/transient ischaemic attack; $\mathrm{X}^{2}$ test: Chi-Square test; Obesity: body mass index (BMI) $>25 \mathrm{mg} / \mathrm{m}^{2}$; New onset: patient's palpitation had occurred within 3 months preceding the first visit

*: patients may have more than one chronic comorbidity

\subsection{Outcomes of Holter Monitoring}

One hundred and nine patients (39.1\%) presented with palpitation of primary care setting were detected by Holter to have significant cardiac arrhythmia. The Holter monitoring defined significant cardiac arrhythmia included 31 cases of prolong QT interval, which accounted for $28.4 \%$ of all significant Holter outcomes. (Table 2) Other significant findings included 25 cases (22.9\%) frequent supraventricular or ventricular ectopy, 14 cases (12.8\%) supraventricular or ventricular ectopy in bigeminy or trigeminy, and 11 cases (10.1\%) of paroxysmal supraventricular tachycardia etc.

Table 2. Frequency of significant cardiac arrhythmia.

\begin{tabular}{llll}
\hline & Frequency & \% of Significant (109) & \% of Total (279) \\
\hline Prolong QT interval & 31 & $28.4 \%$ & $11.1 \%$ \\
SVE/VE (Freq) & 25 & $22.9 \%$ & $9.0 \%$ \\
SVE/VE (Bi/Tri) & 14 & $12.8 \%$ & $5.0 \%$ \\
PSVT & 11 & $10.1 \%$ & $3.9 \%$ \\
AF/PAF & 10 & $9.2 \%$ & $3.6 \%$ \\
Sig. Brady/Tachy & 6 & $5.5 \%$ & $2.2 \%$ \\
SSS & 5 & $4.6 \%$ & $1.8 \%$ \\
Sig. ST change & 5 & $4.6 \%$ & $1.8 \%$ \\
HB $\left(2^{0}\right.$ or $\left.3^{0}\right)$ & 2 & $1.8 \%$ & $0.7 \%$ \\
Total & 109 & $100 \%$ & $39.1 \%$ \\
\hline
\end{tabular}

SVE/VE (Freq): supraventricular ectopy/ventricular ectopy (Frequent); SVE/VE (Bi/Tri): supraventricular ectopy/ventricular ectopy (Bigeminy/Trigeminy); PSVT: paroxysmal supraventricular tachycardia; AF/PAF: atrial fibrillation/Paroxysmal atrial fibrillation; Sig. Brad/Tachy: significant bradycardia/trachycardia; SSS: sick sinus syndrome, HB $\left(2^{0}\right.$ or $\left.3^{0}\right)$ : Heart block (Second or third degree); Sig. ST change: significant ST segment change.

In Table 3, the common significant Holter outcomes were stratified by age. It was clearly observed that PAF/AF and SVE/VE occurred more frequent in elderly, while prolong QT interval was more common among younger patients. 
Table 3. Significant Holter results in patients investigated for palpitations: stratification by age.

\begin{tabular}{|c|c|c|c|c|c|}
\hline Age, years & Significant Outcome & Prolong QT Interval & SVE/VE: (Fre/Bi/Tri) & PSVT & AF/PAF \\
\hline$\leq 40(n=27)$ & $12(44.4 \%)$ & $4(33.3 \%)$ & $1(8.3 \%)$ & $3(25 \%)$ & 0 \\
\hline $41-50(n=51)$ & $13(25.5 \%)$ & $3(23.1 \%)$ & $5(38.5 \%)$ & 0 & 0 \\
\hline $51-60(n=74)$ & $22(29.7 \%)$ & $10(45.5 \%)$ & $7(31.8 \%)$ & $2(9.1 \%)$ & $1(4.5 \%)$ \\
\hline $71-80(n=53)$ & $26(49.1 \%)$ & $5(19.2 \%)$ & $10(38.5 \%)$ & $3(11.5 \%)$ & $4(15.4 \%)$ \\
\hline$>80(n=11)$ & $9(81.8 \%)$ & $1(11.1 \%)$ & $6(66.7 \%)$ & 0 & $2(22.2 \%)$ \\
\hline
\end{tabular}

SVE/VE (Fre/Bi/Tri): supraventricular ectopy/ventricular ectopy (Frequent/Bigeminy/Trigeminy); PSVT: paroxysmal supraventricular tachycardia; AF/PAF: atrial fibrillation/Paroxysmal atrial fibrillation.

\subsection{Patient Characteristics Associated with Cardiac Arrhythmia}

The patient characteristics associated with significant Holter outcome or cardiac arrhythmia were also summarized in Table 1. Using Chi-Square test, it was found that patients who were smoker, aged more than 80 years old, or with concomitant ischaemic heart disease (IHD) were statistically significant associated with significant cardiac arrhythmia $(\mathrm{p}<$ 0.05 ). The odd ratio (OR) of patients with IHD for significant Holter outcome was 11.60 (95\% CI: 1.41 to 95.63), while the OR $(95 \% \mathrm{CI})$ for smokers and patient aged greater than 80 years was $1.84(1.01-3.31)$ and $6.00(1.09$ to 33.02$)$ respectively.

Multivariate analyses by logistic regression procedure were applied to determine the patient characteristics associated with significant Holter outcome. The factors including age, smoke status, and concomitant of chronic disease, hypertension, obesity or ischaemic heart disease were included for analysis. The final fitted model revealed that patients who were smoker, with concomitant IHD were statistically significant associated with significant Holter outcomes. (Table 4) The OR (95\% CI) for smoker is $1.85(1.01-3.37)$, while for IHD is $11.99(1.41-$ 102.13).

Table 4. Factors associated with significant Holter outcomes (Logistic Regression fitted model).

\begin{tabular}{llll}
\hline Variable & OR & $\boldsymbol{p}$-value & $\mathbf{9 5 \%}$ CI \\
\hline Age & 1.01 & 0.121 & $0.99-1.03$ \\
Smoke & 1.85 & 0.046 & $1.01-3.37$ \\
IHD & 11.99 & 0.023 & $1.41-102.13$ \\
Obese & 1.44 & 0.159 & $0.87-2.40$ \\
\hline
\end{tabular}

IHD: ischaemic heart disease

\section{Discussion}

Our retrospective cross-sectional review revealed that 39.1\% of patients presented with palpitation in primary care setting had Holter monitoring confirmed cardiac arrhythmia, with prolong QT interval as the commonest condition detected.

Palpitation or the underlying suspected cardiac arrhythmia is usually non-sustained and of short duration, the standard electrocardiography (ECG) can be entirely normal when those patients presented to doctors. In a case series of 190 consecutive patients presenting with palpitation, the etiology was cardiac in $43 \%$ and psychiatric in $31 \%$ of patients. [12] One study involving 1454 ambulatory elderly in Hong Kong revealed that palpitation were present in $8.3 \%$ while cardiac arrhythmia were found in $12.6 \%$ subjects. [13] Holter monitor was usually used to determine the cause of palpitation, however, the yield of this instrument was low especially in patients whose symptoms occurred infrequently, i.e. 2-24\% of the Holter outcomes were confirmed to be significant. [14-17] However, it strongly depended on the population studied. [18] In Sreekumar study, involving 8,973 Holter recordings done in hospital setting, the diagnostic yield of patients with palpitation was $16.5 \%$. [7] In Summerton study, involving patients with new onset palpitations in general practice, $19 \%$ of patients had significant cardiac arrhythmia. [6] In Chu study, involving patients in a regional Accident and Emergency Department of Hong Kong arranged for Holter monitoring, $16.7 \%$ of patients presented with palpitation were found to have significant cardiac arrhythmia. [11]

Around 2,688 patients underwent Holter monitoring for investigation of palpitation in Sreenkumar study, the commonest two findings were $6.6 \%$ paroxysmal atrial fribrillation (PAF) and 4.4\% frequent ectopics. [7] In our study, prolong QT interval (11.1\%) was the commonest finding, while PAF was the fifth $(3.6 \%)$ and frequent ectopics was the second $(9 \%)$ condition detected.

\subsection{Implications for Practice}

Holter is well accepted as a cost effective and non-invasive investigation with a reasonable yield. In Australia, Holter monitoring can be ordered by general practitioner without specialist review. [19] Holter monitoring is not the routine investigation under primary care setting in Hong Kong. Our study suggested that Holter monitoring in primary care setting enabled an earlier detection of cardiac arrhythmia and speed up the referral and further care by specialist. Further studies may be conducted to investigate the feasibility and cost effectiveness of implementing Holter monitoring in the primary care setting.

\subsection{Strengths and Limitations}

Our study recruits consecutive cases from primary care clinic and arrangement for Holter monitoring are ordered by primary care doctors, therefore, the study population is representative of primary care setting. On the other hand, patients with palpitation are not all indicated for Holter monitoring, a patient selection bias cannot be excluded. This study involves patients from one primary care clinic only, which limits the generalizability of the study results. 


\section{Conclusion}

In this study for patients presented with palpitation in primary care setting, significant cardiac arrhythmia were detected in $39.1 \%$ of Holter monitoring which required referral to specialist for further management. Patients who were smoker, or with concomitant ischaemic heart disease were more likely to have significant cardiac arrhythmia.

\section{Acknowledgment}

The authors would like to thank Tung Wah Group of Hospitals (TWGHs) for supporting this study.

\section{References}

[1] Barsky AJ, Ahern DK, Bailey D, Delamater BA. Predictors of persistent palpitations and continued medical ultilization. $J$ Fam Pract. 1996;42: 465-72. [1].

[2] Lietch J, Klein G, Yee R. Can patients discriminate atrial fibrillation from regular supraventricular tachycardia? $\mathrm{Am} \mathrm{J}$ Cardio. 1991;68: 962-6.

[3] Wexler RK, Pleister A, Raman S. Outpatient approach to palpitation. Am Fam Physician. 2011:84 (1): 63-9.

[4] ACC/AHA Guidelines for Ambulatory Electrocardiography: Executive Summary and Recommendations: A Report of the American College of Cardiology/American Heart Association Task Force on Practice Guidelines. Circulation. 1999;100: 886-93.

[5] java applets for power and sample size. http://www.divms.uiowa.edu/ rlenth/Power/.

[6] Summerton N, Mann S, Rigby A, Petkar S and Dhawan J. New-onset palpitations in general practice: assessing the discriminant value of items within the clinical history. Family Practice. 2001;18: 383-92.

[7] Sreekumar S, Dauda B, Neha S, Abdel S et al. Limited Clinical Utility of Holter Monitoring in Patients with Palpitation or Altered Consciousness: Analysis of 8973 Recordings in 7394 Patients. A. N. E. 2008;13 (1): 39-43.
[8] Khamis RY, Dancy M. Palpitations. Medicine. 2008;37 (2): 100-5.

[9] Fuster V, Ryden LE, Cannom DS et al. ACC/AHA/ESC 2006 guidelines for management of patients with atrial fibrillation-executive summary. J Am Coll Cardiol. 2006;48 (4): 854-906.

[10] Arya A, Haghjoo M, Khosrawi A, Emkanjoo Z, Sadr-Ameli MA. Predictors of arrhythmic events during second day monitoring in patients with normal first day Holter recordings. Indian Heart J. 2005;57: 241-4.

[11] Chu CKK, Lee EFT, Leung CS, Lit ACH. The use of ambulatory electrocardiography in the emergency medicine ward to assess patients with symptoms possibly related to cardiac arrhythmia: a sharing of experience in a local hospital. Hong Kong Journal of Emergency Medicine. 2010;Vol. 17 (4): 316-322.

[12] Weber BE, Kapoor WN. Evaluation and outcomes of patients with palpitations. Am J Med. 1996;100 (2): 138-48.

[13] Lok NS, Lau CP. Prevalence of palpitations, cardiac arrhythmias and their associated risk factors in ambulant elderly. Int J Cardiol. 1996;54: 231-6.

[14] DiMarco JP, Philbrick JT. Use of ambulatory electrocardiographic (Holter) monitoring. Ann Inter Med. 1990;113: 53-68.

[15] Safe AF, Maxwell RT. Transtelephonic electrocardiographic monitoring for detection and treatment of cardiac arrhythmia. Postgrad Med J. 1990;66: 110-2.

[16] Thomas LE, Shapiro LM, Perrins EJ, Fox KM. Detection of arrhythmia: limited usefulness of patients activated recording devices. Br J Med (Clin Res Ed). 1984;289: 1106-7.

[17] Scalvini S, Zanelli E, Martinelli G, Baratti D, Giordano A, Glisenti F. Cardiac event recording yields more diagnosis than 24-hour Holter monitoring in patients with palpitations. $J$ Telemed Telecare. 2005;11 (supp 1): 14-6.

[18] Seipel L. The clinical value of Holter ECG recording. Internist. 2004;45: 1035-41.

[19] Mclellan A, Mohamed U. Ambulatory electrocardiographic monitoring. Australian Family Physician. 2011;40 (8): 596-8. 